\title{
A Single Pathology Specialty Service for Hyperthyroid Patients improves care and outcomes compared to general endocrine clinics - results and implications of an audit: re-audit cycle of clinical outcomes for differing hyperthyroid care models.
}

\section{Wong ML, Olateju T, Munday J, Cummings MH, Meeking DR, Cranston ICP Queen Alexandra Hospital, Portsmouth Hospitals NHS Trust, Cosham, Portsmouth PO6 3LY}

\section{Introduction}

-Prior to May 2011, we ran 4 separate consultant-led endocrine services with 6-8 new patient referrals per week with hyperthyroidism.

-Patients were seen "ad hoc" in general endocrine clinics

-Concern raised regarding timeliness of care

\section{Audit}

-203 patients under active follow-up (FU) (active =on anti-thyroid medications or within 6 months of radioactive iodine)

-Audit aim: to determine care received by patients with thyrotoxicosis and time interval between outpatient clinic appointments (OPAs)

\section{Standards}

Based on UK guidelines for use of TFTs ${ }^{1}$

1) All patients with abnormal thyroid function under treatment should be seen within 6 weeks (+/-7days)

2) All euthyroid patients under treatment should be seen within 3 months (+/- 7days).

\section{Intervention}

-Introduction of dedicated "weekly multidisciplinary clinic" hyperthyroid service to streamline care and allow for appropriate followup as well as opportunity for liason with other specialists for those who require it.

\section{Reference}

1. UK guidelines for use of TFTs July 2006 (Association for Clinical Biochemistry, British Thyroid Association,

British Thyroid Foundation)
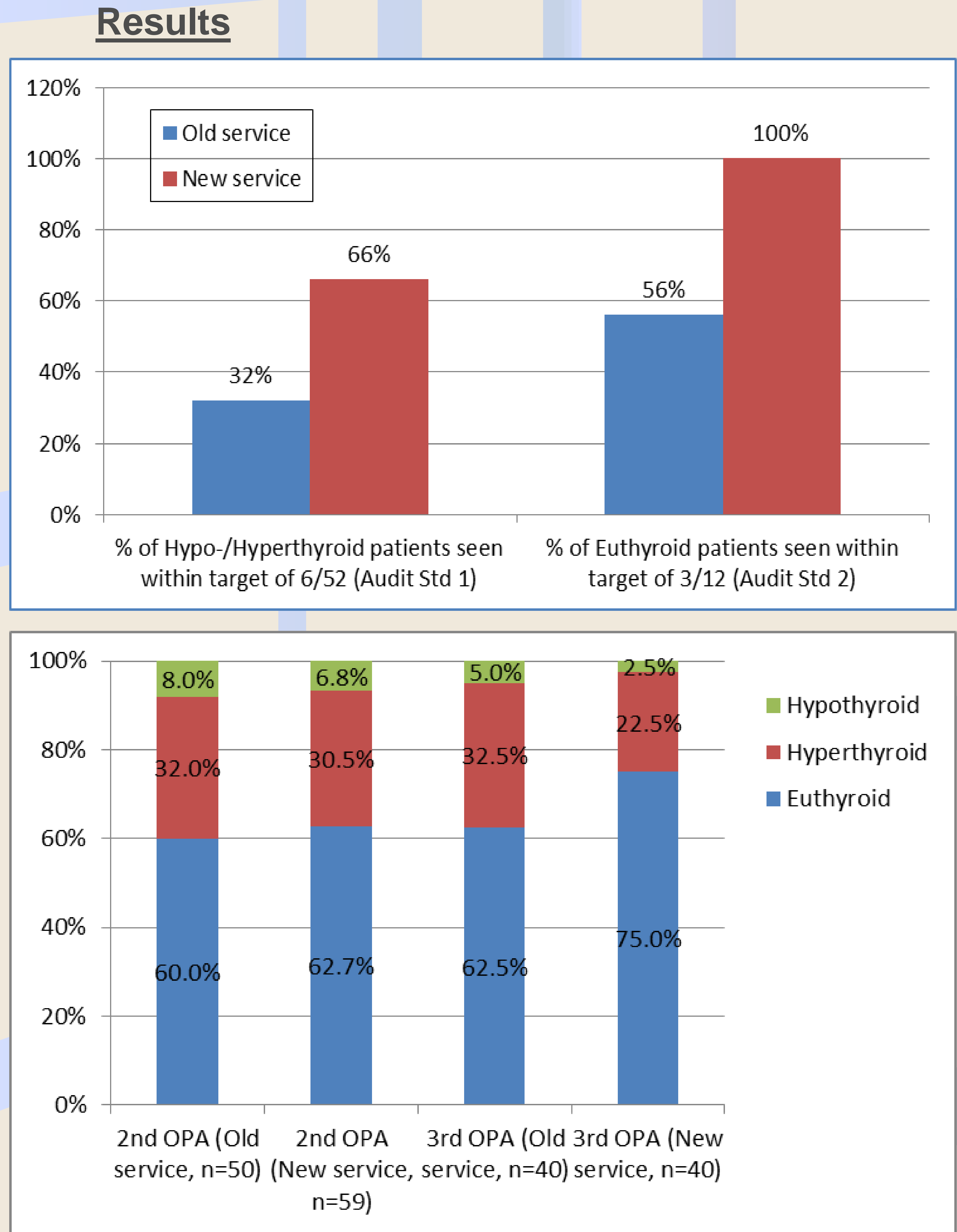

- Mean FU duration was 7 weeks (new service) vs 20 weeks (old) for those with abnormal TFTs

- Mean FU duration was 8 weeks (new service) vs 30 weeks (old) for euthyroid patients

- $66 \%$ attended all 3 OPAs (new service) compared to $44 \%$ (old service)

\section{Conclusions}

- Dedicated hyperthyroid services improved quality of care and outcomes for patients

-There was better use of services and resources -More patients seen within target standards and euthyroid status achieved earlier.

-Significant reduction in re-appointment and nonattendance rates. 